\title{
De la fisiología al ventilador, interacción corazón pulmón durante la ventilación mecánica
}

From physiology to ventilator, heart lung interaction during mechanical ventilation

Da fisiologia a ventilador, interação coração pulmão durante ventilação mecânica

Humberto Ochoa Salmorán, " Ivonne Martínez Martínez,

Karla Sharon Lugo Bautista, ${ }^{*}$ Enrique Juan Díaz Greene*

\section{RESUMEN}

La interacción corazón-pulmón fue observada por Hales desde el siglo XVIII. Este sistema funciona de forma simple como un circuito y una bomba. Al ocupar un mismo espacio físico: la caja torácica; los cambios de presión dentro de ésta afectarán el sistema formado por el corazón y el pulmón. Durante el ciclo respiratorio, la presión torácica varía, afectando el gradiente de presión de sangre que entra y sale del tórax. Cada uno de los componentes de la precarga como de la postcarga deben estudiarse por separado, para posteriormente entender la interdependencia del ventrículo derecho como izquierdo y su repercusión en la circulación pulmonar. La ventilación con presión positiva eleva la presión intratorácica, condiciona la disminución del llenado del ventrículo derecho, condiciona un aumento de la postcarga del mismo y reduce el flujo sanguíneo pulmonar. Todos los cambios que se presentan durante la ventilación mecánica pueden desencadenar en inestabilidad hemodinámica. El ventrículo derecho, al tener sus resistencias vasculares y precarga reducidas, está sometido particularmente a estos cambios. El volumen tidal disminuido, entre otras estrategias, se emplea con la finalidad de reducir los efectos mecánicos que sufre el ventrículo derecho.

Palabras clave: Ventilación, mecánica, interacción, cardiopulmonar, fisiología.

\section{ABSTRACT}

The heart-lung interaction was observed by Hales since the 18th century. This system works simply as a circuit and a pump. By occupying the same physical space: the rib cage, changes in pressure within it will affect the system formed by the heart and lung. During the respiratory cycle the thoracic pressure varies, affecting the blood pressure gradient that enters and leaves the chest. Each of the components of the preload and afterload should be studied separately. To later understand the interdependence of the right ventricle as left and its impact on the pulmonary circulation. Ventilation with positive pressure increases intrathoracic pressure, determines decreased filling of the right ventricle, conditioning an increase in its afterload and decreasing pulmonary blood flow. All changes that occur during mechanical ventilation can trigger hemodynamic instability. The right ventricle having its reduced vascular resistance and preload is particularly subject to these changes. The use of a reduced tidal volume among other strategies, are used in order to reduce the mechanical effects suffered by the right ventricle.

Keywords: Ventilation, mechanical, interaction, heart lung, physiology.

\section{RESUMO}

A interação coração-pulmão foi observada por Hales desde o século XVIII. Este sistema funciona simplesmente como um circuito e uma bomba. Por ocupar o mesmo espaço físico: a caixa torácica, as mudanças de pressão dentro dela afetarão o sistema formado pelo coração e pulmão. Durante o ciclo respiratório, a pressão torácica varia afetando o gradiente de pressão do sangue que entra e sai do tórax. Cada um dos componentes de pré-carga e pós-carga deve ser estudado separadamente. Para posteriormente entender a interdependência dos ventrículos direito e esquerdo e seu impacto na circulação pulmonar. A ventilação com pressão positiva aumenta a pressão intratorácica, diminui o enchimento do ventrículo direito, condiciona o aumento da sua pós-carga e diminui o fluxo sanguíneo pulmonar. Todas as alterações que ocorrem durante a ventilação mecânica podem levar à instabilidade hemodinâmica. $O$

\section{* Hospital Ángeles Pedregal. Ciudad de México, México.}

Recepción: 20/12/2019. Aceptación: 16/07/2020.

Citar como: Ochoa SH, Martínez MI, Cabrera PD, Lugo BKS, Díaz GEJ. De la fisiología al ventilador, interacción corazón pulmón durante la ventilación mecánica. Med Crit. 2020;34(5):283-292. https://dx.doi.org/10.35366/96459

www.medigraphic.com/medicinacritica ventrículo direito, tendo sua resistência vascular e pré-carga reduzidas, está particularmente sujeito a essas alterações. A utilização da diminuição do volume corrente, entre outras estratégias, é utilizada para diminuir os efeitos mecânicos sofridos pelo ventrículo direito.

Palavras-chave: Ventilação, mecânica, interação, cardiopulmonar, fisiologia.

\section{INTRODUCCIÓN}

La interacción corazón pulmón fue demostrada en 1733 por Hales y colaboradores al observar la variación de la circulación en el ciclo respiratorio en un manómetro de cristal insertado en la arteria carótida de un caballo. Esto refleja la interrelación entre los sistemas respiratorio y cardiovascular durante cada uno de sus ciclos. En la segunda mitad del siglo XIX la presión pleural fue reconocida como un fenómeno de la interacción cardiopulmonar. Posteriormente, en la Segunda Guerra Mundial, la necesidad de aportar oxígeno con presión positiva a grandes alturas en los pilotos de combate llevó a un entendimiento cada vez más profundo de la interacción corazón pulmón explicado de forma simple, el sistema circulatorio tiene dos componentes: el circuito y la bomba. Para entender la interacción corazón pulmón en la ventilación mecánica es necesario conocer los cambios durante la respiración espontánea por medio del ejercicio. En éste se consume oxígeno que requiere un aumento del flujo sanguíneo y produce dióxido de carbono. ${ }^{1-4}$

Corazón y pulmón ocupan un espacio físico en la caja torácica, interconectados por los vasos sanguíneos, «el corazón es una cámara de presión dentro de una cámara de presión». Los cambios en la presión torácica afectarán los sistemas de presión dentro del corazón y de éste a los espacios extratorácicos, pero no se afectan las relaciones vasculares intratorácicas. Esto se entiende porque el flujo a través del circuito es determinado por el gradiente de presiones dentro del propio circuito. ${ }^{1}$ La presión torácica varía durante el ciclo respiratorio y durante las maniobras de Valsalva y Müller, afectando el gradiente de presión entre la sangre que entra al tórax, conocido como retorno venoso, y la sangre que sale del tórax o volumen expulsivo del ventrículo izquierdo. ${ }^{3}$ El gradiente de presión del flujo sanguíneo es diferente para los lados arterial y venoso de la circulación. En el retorno venoso el gradiente de presión es dado por la presión atmosférica y la presión abdominal y por los reservorios sistémicos me- 
dios, denominados presión de llenado media circulatoria, a la aurícula derecha y para el circuito arterial desde el ventrículo izquierdo hasta el árbol arterial (Figura 1). Así los cambios en la presión intratorácica durante la ventilación producirán cambios en la precarga, postcarga y la contractilidad al afectar el tamaño, presión y disposición de las cámaras cardiacas. ${ }^{1-3,5}$

Dado que la presión intratorácica es la presión circundante para el corazón, la presión auricular derecha relativa al llenado ventricular derecho (VD) se cuantifica mejor como la presión auricular derecha menos la presión intratorácica, y se denomina presión transmural. Una presión positiva distiende la cavidad y una negativa la colapsa. ${ }^{1,3}$ Del mismo modo la presión de eyección del ventrículo izquierdo (VI) es estimada como la presión arterial menos la presión intratorácica. Aunque estas mediciones varían de acuerdo con la presión intratorácica, se sabe que tanto la presión de llenado media circulatoria como la presión arterial no se ven afectadas por cambios aislados de la presión intratorácica. Por tanto, los cambios en esta última pueden alterar los gradientes de presión mediante la alteración cíclica de la presión auricular derecha y presión transmural del VI, ambas durante la respiración o en el estado estable si la presión intratorácica se mantiene o aumenta con relación a la atmósfera. Sin embargo, el gradiente de presión de la arterial pulmonar a la aurícula izquierda (Al) no se ve alterado por los cambios en la presión intratorácica porque todo el circuito está dentro del tórax. Así la circulación sistémica se puede ver afectada por los cambios en la presión intratorácica, mientras que la circulación pulmonar no se ve afectada, a menos que los volúmenes pulmonares cambien también. ${ }^{1} \mathrm{EI}$ objetivo del presente trabajo es conocer y entender la interacción corazón pulmón durante el ejercicio y la ventilación mecánica para optimizar las estrategias de esta última de acuerdo con el contexto clínico del paciente evitando disminuir el gasto cardiaco.

\section{CORAZÓN DERECHO Y DETERMINANTES DE LA PRECARGA}

La precarga corresponde al largo relativo de la fibra muscular previo a su contracción, se define como el estrés en la pared del ventrículo derecho al final de la diástole, momento en el cual el VD está lleno. El volumen al fin de la diástole en el VD está determinado por el retorno venoso hacia la aurícula derecha (AD), y es proporcional al gradiente de presión entre el sistema extratorácico y la AD. Por otro lado, los cambios en la presión intratorácica afectan el retorno venoso hacía la $A D$ al modificar la presión transmural. Durante la inspiración espontánea, disminuye la presión intratorácica, por tanto, eleva la presión transmural de la $\mathrm{AD}$, causando que ésta se distienda, su presión cae y de esta manera aumenta el retorno venoso. Un factor extrapulmonar que aumenta el retorno venoso, es que el retorno de sangre se incrementa desde la vena cava inferior dada la elevación de la presión intraabdominal durante la inspiración, producto del descenso del diafragma, disminuyendo la capacitancia de los vasos abdominales, y aumentando el gradiente de presión desde la vena cava inferior. El incremento del retorno venoso no es lineal ni infinito; cuando la presión intratorácica llega a un valor muy negativo éste se transmite a la $A D$ y a las venas que entran al tórax, provocando un colapso de las venas a la entrada del tórax al hacerse muy negativa su presión transmural, limitando así el flujo venoso. Por otra parte, una elevación de la presión de la $A D$ determinará una disminución del retorno venoso, cuya caída será mayor al acercarse a la presión media sistémica. Estas condiciones se modifican de acuerdo con el estado del paciente; así la pendiente de retorno venoso presentará un incremento en situaciones de disminución de la resistencia o aumento de la volemia y reducción de la pendiente del retorno venoso al bajar la volemia o aumentar las resistencias, lo cual queda graficado en la curva de retorno venoso (Figura 2).3,6

En reposo, el volumen al final de la diástole del VD varía en un amplio rango con cambios mínimos en la presión transmural del VD. Bajo condiciones de reposo la fuerza de eyección del VD es constante, esto explica por qué la fluidoterapia incrementa el volumen del VD al final de la sístole al igual que el volumen al final de la

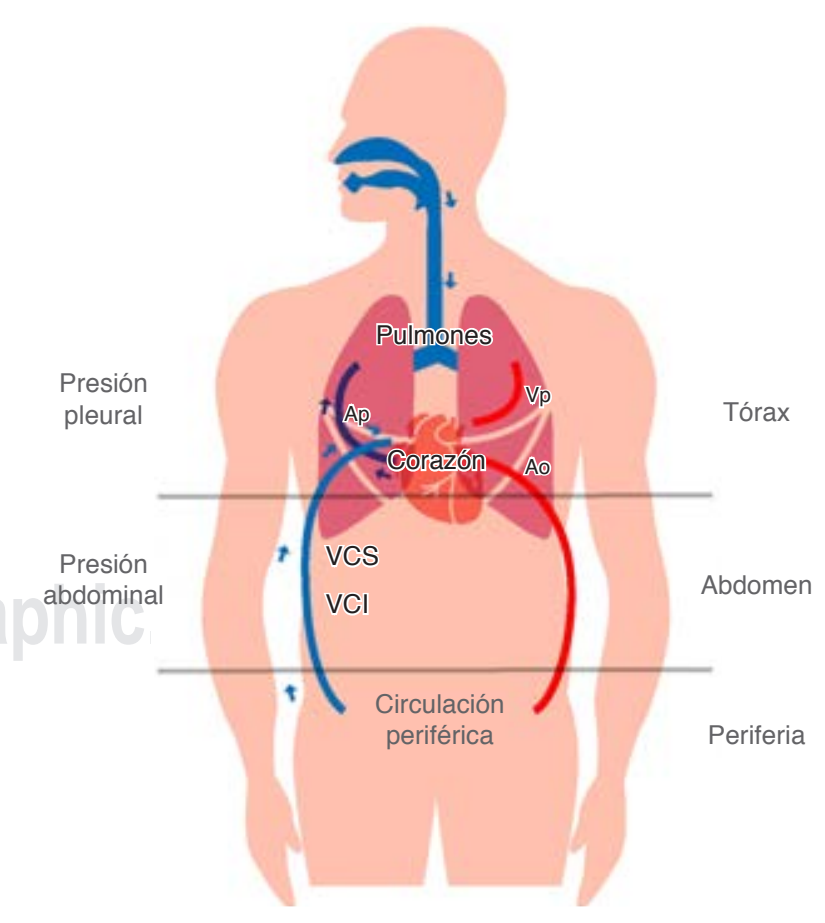

Figura 1: Modelo de compartimentos de la circulación. VCS = vena cava superior; $\mathrm{Ap}=$ arteria pulmonar, $\mathrm{Vp}=$ venas pulmonares; $\mathrm{Ao}=$ aorta; $\mathrm{VCl}=$ vena cava inferior. 


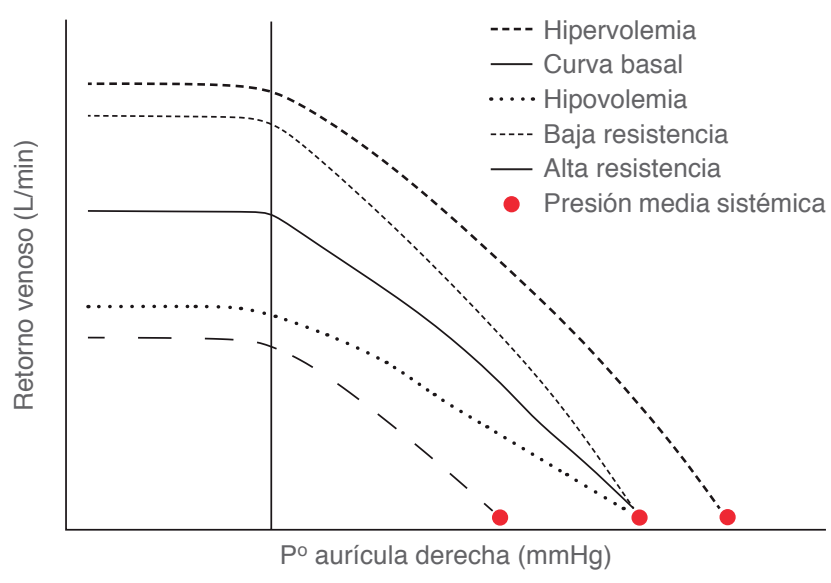

Figura 2: Curva de retorno venoso y sus efectos en la resistencia o volemia.

diástole. Sin embargo, el gradiente de presión para el retorno venoso al VD de la circulación es la presión de llenado media circulatoria relativa a la presión auricular derecha. El sistema venoso transporta $70 \%$ del volumen sanguíneo en el cuerpo, mucho de este volumen se aloja en los vasos que censan la presión alrededor de ellos y la presión atmosférica. Si se retirara la sangre de la circulación adinámica, se vería que la presión de llenado media circulatoria disminuiría a cero a pesar de tener más de la mitad del volumen todavía en la circulación. Esto se da porque la cantidad de sangre en la circulación por debajo del volumen que causa incremento en la presión vascular, Ilena el espacio intravascular no por el tramo vascular, pero sí causa una expansión conformacional de un estado colapsado. Una vez distendida, cualquier aumento en el volumen intravascular provocará un incremento de presión de llenado media circulatoria. La cantidad de sangre en la circulación sistémica desde este punto de inflexión es llamada volumen sin estrés de la circulación y la cantidad por arriba de éste se llama volumen de estrés. Cualquier incremento del volumen intravascular por encima del punto del volumen de estrés resultará en una elevación en la presión de llenado media circulatoria a lo largo de la relación de la complianza venosa. Así, la presión de llenado media circulatoria es determinada por el volumen de estrés causando una presión transmural positiva, contra la pared vascular. En resumen, el volumen de sangre total circulante define tanto el volumen de estrés como el de no estrés. El retroceso elástico contra estos vasos sanguíneos provee la fuerza suficiente de presión para llevar la sangre de regreso al corazón. Los diferentes lechos vasculares tienen diferentes volúmenes de no estrés y alteran la distribución del flujo sanguíneo hacia los circuitos de bajo volumen de no estrés, o incrementan el tono vasomotor como los vasopresores, los que incrementarán la presión de llenado media circulatoria, esto al aumentar la presión del flujo para el retorno ve- noso. Por último, el ciclo respiratorio mediante la alteración selectiva de la presión auricular derecha puede influir también en este gradiente de presión. ${ }^{1,7}$

La presión auricular derecha es la presión opuesta al retorno venoso, que se opone a la presión de llenado media circulatoria. El cambio dinámico en la presión auricular derecha durante el ciclo de ventilación causa cambios recíprocos en las tasas de flujo venoso. Se pensaba que la presión auricular derecha estaba influenciada por la complianza de la AD y por los cambios en la presión transmural durante los ciclos respiratorios; así, cuando existe falla del VD y dilatación de la AD, pequeños cambios en el volumen intravascular inducirán aumentos proporcionales en la presión auricular derecha. La presión transmural para la AD se considera el gradiente entre la presión auricular derecha y la presión que rodea el exterior del miocardio. En ausencia de cardiopatía como tamponade cardiaco, ésta será la presión pleural. Durante la inspiración, la presión pleural disminuye para una presión auricular derecha constante transmural, lo que causa que la presión auricular derecha baje. Esto provoca un aumento inmediato en el retorno venoso, lo que eleva el volumen diastólico final del VD y el volumen del VD en el siguiente latido. Por otro lado, en la espiración la presión pleural es menos negativa, con lo que la presión auricular derecha aumenta hasta su valor espiratorio final y el retorno venoso disminuye ligeramente. . $^{8-10}$

Mientras el efecto de la presión intratorácica en la presión auricular derecha está bien documentado, el rol de la presión transmural de la presión auricular derecha en el rendimiento del VD es cuestionable. Tyberg y colaboradores midieron la presión pericárdica y la presión auricular derecha en pacientes sometidos a cirugía cardiaca encontrando que ambas aumentaban de forma equivalente, de modo que la presión auricular derecha transmural permanecía constante. De forma similar Lansdorp y su equipo midieron las presiones pleurales yuxtacardiacas y pericárdicas empleando globos insuflados con aire en 20 pacientes postcolocación de bypass con diferentes volúmenes tidales durante la ventilación mecánica, encontrando que la presión auricular derecha transmural (derivada de la presión auricular derecha menos la presión pericárdica) no cambiaba con el incremento del volumen tidal desde 4, 6, 8 y 10 $\mathrm{mL} / \mathrm{kg}$. Ambos estudios concuerdan con la afirmación de que, en rango fisiológico, el llenado del VD se produce por debajo de su volumen de no estrés. ${ }^{1,9,11}$

La ventilación con presión positiva revierte el efecto en la presión auricular derecha durante el ciclo respiratorio, aumentando la presión auricular derecha durante la inspiración y disminuyendo durante la espiración. Las presiones en la vía aérea, presión auricular derecha, pericárdicas y pleurales se incrementan con el aumento del volumen tidal y la presión intratorácica de forma li- 
neal. Con la inflación mecánica de los pulmones durante la inspiración, la presión intratorácica y la presión auricular derecha se elevan. Esto disminuye la diferencia de presión para el retorno venoso y el volumen al final de la diástole del VD. ${ }^{11,12}$

Este concepto de cómo la PIT altera el gradiente de presión para el RV, y por lo tanto el gasto cardiaco, es vital para los clínicos, sobre todo en el contexto de pacientes hipovolémicos, el gradiente de presión que conduce la sangre desde los lechos venosos al corazón es de 4-8 mmHg. Dado que la resistencia del retorno venoso es muy baja, un gradiente de presión pequeño es adecuado para llevar $100 \%$ del gasto cardiaco de regreso al corazón cada minuto. Por lo tanto, aumentos pequeños de la presión espiratoria final positiva (PEEP) causan una reducción relativamente grande de la precarga y del gasto cardiaco en general. Este efecto puede mitigarse al incrementar la presión de llenado media circulatoria, el volumen de estrés o el tono vascular. Katira y colaboradores demostraron que al emplear un volumen tidal elevado y PEEP de cero ante disminuciones bruscas de la presión auricular derecha, el volumen al final de la diástole del VD produce una elevación de la presión de eyección del VD (aumento de la resistencia vascular pulmonar por gran volumen tidal), desarrollando cor pulmonale de forma progresiva. Simplemente al agregar $10 \mathrm{cmH}_{2} \mathrm{O}$ de PEEP y volumen tidal bajo, estos efectos perjudiciales se reducen. ${ }^{13-15}$

Además de su efecto directo en el corazón, tanto la presión negativa como positiva afectan la precarga incrementando el retorno venoso desde la vasculatura abdominal por la elevación de la presión abdominal debido a la excursión diafragmática. En el caso de la ventilación con presión negativa, la cantidad de sangre que regresa al corazón está limitada por la propia presión intratorácica cuando la presión auricular derecha se vuelve subatmosférica, lo que causa que los grandes vasos colapsen, pues ingresan a través de la entrada torácica creando un segmento de flujo limitado. ${ }^{1}$ Durante la ventilación mecánica es importante mitigar la disminución en la precarga del VD causada por una elevación de la presión intratorácica al aumentar la presión de llenado media circulatoria y por lo tanto, minimiza los efectos perjudiciales del incremento de la presión auricular derecha en el gradiente de presión para el retorno venoso. Como se demostró en 42 pacientes postoperados reanimados con soluciones intravenosas se utilizó una pausa inspiratoria de 25 segundos y PEEP de $20 \mathrm{mmHg}$. Con esto el gasto cardiaco se mantuvo sin cambios durante la pausa inspiratoria y aumento progresivo de la PEEP pese a la elevación de la presión auricular derecha, ya que la presión intraabdominal se incrementó en una cantidad similar, permitiendo que los compartimientos venosos intraabdominales aumentaran proporcionalmente sus presiones venosas ascendentes. ${ }^{1,16}$

\section{CORAZÓN IZQUIERDO Y DETERMINANTES DE LA PRECARGA}

La respiración puede afectar la precarga del VI a través de varios mecanismos desde los cambios que se generan en el VD hasta en la distensibilidad de ambos ventrículos. Durante la inspiración la presión intratorácica disminuye, condicionando un aumento del retorno venoso y llenado del VD, con un incremento de la resistencia vascular pulmonar producto del aumento del volumen pulmonar, determinando además una reducción del flujo de salida de sangre del VD. Al ser éste muy distensible se produce un desplazamiento del septum interventricular a la izquierda, lo que determina una disminución de la capacidad neta del VI y de su llenado, en especial por ser el VI poco distensible, rodeado en su pared libre por pericardio fijo y por el pulmón. Además, el decremento de la presión intratorácica determina en forma momentánea un aumento del pool sanguíneo de los vasos pulmonares, y sumado al incremento de la resistencia vascular pulmonar, favorecerá un flujo menor de sangre desde las venas pulmonares hacia la Al y VI. Durante la inspiración el efecto neto es la disminución del volumen de llenado del $\mathrm{VI}$, afectando por tanto su volumen expulsivo. ${ }^{3}$

\section{CORAZÓN IZQUIERDO Y DETERMINANTES DE LA POSTCARGA}

La postcarga es la fuerza que resiste la eyección ventricular. EI VI impulsa sangre desde el tórax a las arterias extratorácicas. Al contraerse el VI crea un gradiente entre él y el espacio yuxtacardiaco, esta presión representa la postcarga del VI. En ausencia de valvulopatía aórtica, esta fuerza de resistencia se determina por la presión aórtica, la elastancia arterial y la resistencia arterial general. La presión transmural del VI se calcula como la diferencia del peak de presión ventricular y la presión intratorácica. ${ }^{1,3}$ Durante la inspiración espontánea se produce una caída de la presión intratorácica, condicionando una elevación de la presión transmural del VI y, por lo tanto, un incremento de la postcarga del VI. Al elevar la presión intratorácica durante la espiración se produce una disminución de la postcarga del VI, determinando un aumento del volumen expulsivo (Figura 3). ${ }^{4}$ Esto explica la aparición del pulso paradójico en ciertas patologías. En caso de hipertensión crónica o calcificación aórtica, el aumento de la elastancia resultante incrementa la postcarga del VI y altera el volumen sistólico del VI en respuesta al ejercicio y aumenta la mortalidad a largo plazo., ${ }^{1,3}$

Durante la ventilación con presión negativa, la inspiración conduce al decremento de la presión pleural y al incremento de la presión transmural del VI y de eyección del VI. Esto dificulta la contracción del VI por el aumento de la postcarga del VI, haciendo que el volumen sistólico 


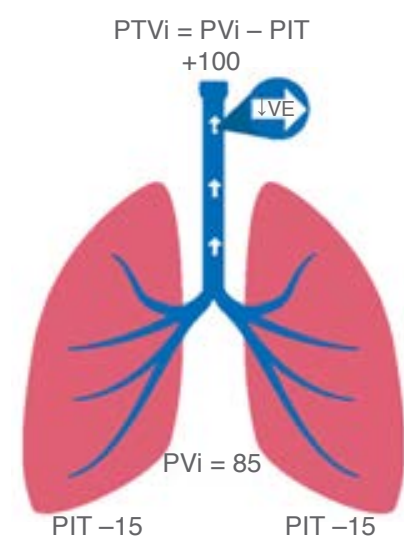

Inspiración espontánea

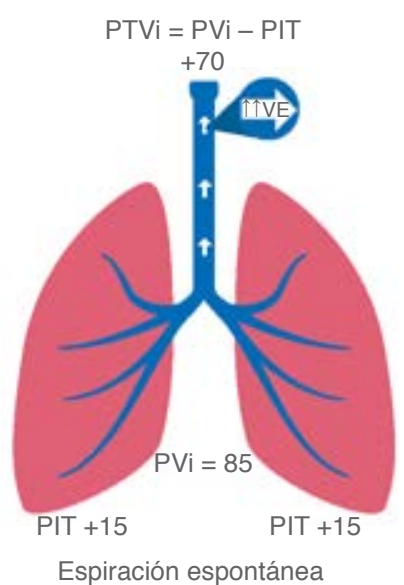

Figura 3: Cambios en el volumen expulsivo durante la inspiración y espiración secundarios a los cambios de la presión transmural del VI.

final del VI se eleve en el primer latido. Lo contrario aplica para la espiración, donde la elevación de la presión intratorácica y de la presión pleural y el decremento de la presión transmural reducen la postcarga al bajar el volumen sistólico final del VI para la misma presión arterial. Sin embargo, si el decremento de la presión intratorácica es marcado, como en el caso de obstrucción de la vía aérea superior, edema laríngeo, apnea obstructiva del sueño o tumores del sistema nervioso central o cervicales, se produce la inspiración en una vía aérea cerrada y la presión intratorácica disminuye de manera importante. Esto causa de forma inmediata grandes aumentos en la postcarga del VI y el retorno venoso, lo que incrementa el contenido de líquido intratorácico, y si es grave y prolongado, promueve el edema pulmonar. ${ }^{1,17}$

Durante la ventilación mecánica, particularmente al emplearse una PEEP o volumen corriente altos durante la inspiración, aumenta la presión pleural, disminuye la presión transmural del VI y reduce la postcarga del VI, ayudando a la eyección del VI incluso si la presión arterial también aumenta. Esto es especialmente notable en pacientes con falla cardiaca congestiva. Sin embargo, estos efectos incrementados del volumen en el VI están limitados por la disminución asociada en el retorno venoso como se describió anteriormente. Además, si el volumen pulmonar aumenta, la resistencia vascular pulmonar también eleva la impedancia de la eyección del VD. Por lo tanto, la combinación del aumento de la presión intratorácica incrementa el gradiente de presión para el retorno venoso, además del aumento inducido por el volumen pulmonar en la resistencia vascular pulmonar puede generar un gasto cardiaco críticamente bajo. Aun así, este efecto de la ventilación con presión positiva es útil en ciertos estadios de enfermedad como insuficiencia cardiaca sistólica izquierda, especialmente si se minimizan los aumentos de volumen pulmonar. Una causa probable de falla del VI durante el destete del ventilador puede ser el incremento asociado de la postcarga del $\mathrm{VI}$ inducido por las disminuciones fásicas de la presión intratorácica con cada respiración espontánea con su aumento obligatorio en el consumo de $\mathrm{O}_{2}$ miocárdico. Este fallo del VI asociado al destete de la ventilación mecánica puede ser la causa principal del fracaso del destete en pacientes críticamente enfermos con ventilación mecánica. ${ }^{18}$

\section{CORAZÓN DERECHO, VOLÚMENES PULMONARES Y DETERMINANTES DE LA POSTCARGA DEL VD}

El corazón derecho se ha descrito más como generador de flujo que como generador de presiones debido a la expulsión a una presión más baja en una vasculatura pulmonar con más complianza. La postcarga del VD se relaciona con la resistencia al flujo de salida de sangre del ventrículo, dicho de otra forma, la postcarga es la tensión o estrés sistólico de la pared ventricular, correspondiendo a la carga en contra, en la cual el músculo ejerce su fuerza contráctil; en vivo corresponde a la resistencia contra la cual la sangre es expulsada. La respiración puede afectar la resistencia vascular pulmonar debido al pH sanguíneo, la $\mathrm{PaO}_{2}$ y de manera importante por el cambio de los volúmenes pulmonares. ${ }^{1,3}$

La resistencia vascular pulmonar depende de la suma de la resistencia de los vasos alveolares (presente en los septos que separan los alveolos adyacentes) y de los vasos extraalveolares (que se encuentran en el intersticio). De esta manera, en la medida en que el volumen pulmonar disminuye, en especial por debajo de la capacidad residual funcional, se produce una baja del radio de los vasos extraalveolares, lo que determina un aumento de la resistencia vascular pulmonar. Esto se suma a la resistencia de los vasos alveolares, los cuales, pese a que los volúmenes pulmonares bajos disminuyen su resistencia, el efecto total es una suma de ambas. Por el contrario, al existir volúmenes pulmonares altos, ya sea por una inspiración normal o forzada, aumentará la resistencia vascular pulmonar total secundaria al incremento de la resistencia de los vasos alveolares, la que se suma a la resistencia disminuida de los vasos extraalveolares, determinada esta última por el decremento de la presión a nivel de intersticio. Así, durante el ciclo respiratorio, en la inspiración se eleva el volumen pulmonar observándose un aumento de la resistencia vascular pulmonar, incrementando de esta manera la postcarga del VD, durante la espiración se observará el efecto contrario, en especial si el volumen pulmonar disminuye. ${ }^{3}$ Los cambios en los volúmenes pulmonares no sólo afectarán las resistencias vasculares pulmonares, sino también los flujos sanguíneos pulmonares. . $^{3,19}$

Durante la ventilación mecánica los cambios en la presión intratorácica son los determinantes de los cambios en la postcarga del VI. Sin embargo, para el VD 
estos cambios tienen efectos mínimos porque toda la vasculatura pulmonar se encuentra en el compartimiento intratorácico y se ve afectada igualmente al cambiar la presión intratorácica. Sin embargo, el cambio en el volumen pulmonar asociado con la ventilación mecánica puede alterar la resistencia vascular pulmonar y la elastancia así como las presiones arteriales pulmonares debido al cambio en las condiciones zonales, todos éstos son determinantes de la postcarga del VD. ${ }^{18,20}$

Durante la inspiración, el aumento del volumen pulmonar hace que la vasculatura pulmonar se distienda, incrementando su complianza y minimizando el aumento de volumen de los movimientos del VD inducidos por la postcarga del VD. Debido a que el VD tiene menos reserva contráctil que el VI, los cambios en la presión intratorácica y la postcarga durante el ciclo respiratorio tienen un efecto mayor en el VD. Este concepto es importante en estadios de enfermedad como el síndrome de dificultad respiratoria aguda (SDRA), donde la vasoconstricción hipóxica puede incrementar la postcarga y causar falla del VD. Las maniobras de reclutamiento alveolar reducirán la impedancia y la resistencia vascular pulmonar en general, promoviendo la eyección efectiva del VD. ${ }^{18,21}$

En la ventilación mecánica, la sobreinflación del volumen pulmonar asociada con la inspiración elevará la resistencia vascular pulmonar y aumentará la postcarga del VD. Por lo tanto, la eyección del VD puede impedirse durante la inspiración si se utilizan grandes volúmenes tidales. Este efecto parece ser mitigado al emplear un volumen tidal de protección pulmonar más bajo del empleado habitualmente y con el uso más reducido de PEEP. Los cambios en la presión pleural afectan las zonas pulmonares de West (Figura 4). Durante la inspiración en la ventilación mecánica, la presión pleural positiva puede crear más áreas de las zonas 1 y 2 , alterando el flujo sanguíneo pulmonar a las áreas de la zona 3, aumentando la resistencia y la postcarga del VD y provocando una elevación de la ventilación del espacio muerto y un posible incremento de los shunt del flujo sanguíneo. Este efecto se debe a los aumentos en el volumen pulmonar causados por la respiración con presión positiva, no a los aumentos de la presión alveolar en sí. Por ejemplo, si el volumen tidal es constante, pero la complianza de la pared torácica se reduce, no se producen cambios en el flujo sanguíneo pulmonar (por ejemplo, SDRA) y los efectos del aumento de la presión alveolar a menudo se mitigan. Sin embargo, el SDRA generalmente se asocia con una elevación de la presión de la arteria pulmonar independientemente de las estrategias de ventilación, por lo que la postcarga del VD todavía puede incrementarse debido a la vasoconstricción hipóxica del edema y la lesión pulmonar en lugar de una presión alveolar alta en relación con la presión de la arteria pulmonar. ${ }^{18}$

\section{VD Y VI ENLACE DINÁMICO: INTERDEPENDENCIA}

EI VD y el VI bombean sangre en paralelo, pero también están conectados en serie. Por lo tanto, el volumen diastólico del extremo del VI se correlaciona con la precarga del VD. La interdependencia ventricular se produce en virtud de los ventrículos que comparten el septum, su ubicación dentro de un espacio pericárdico de volumen fijo y su orientación anatómica de las interasociaciones de miofibrillas de la pared libre. EI VI tiene una forma esférica gruesa con orientación helicoidal, mientras que el VD está envuelto alrededor de la izquierda con una pared libre delgada. Las fibras miocárdicas del VI contribuyen mayormente al septum. La función sistólica del VD depende de este tabique y de la pared libre del VD a las conexiones de fibra de la pared libre del VI. Éste es el caso de la disfunción cardiovascular asociada a la disincronía ventricular (ejemplo, estimulación ventricular única, bloqueos de rama o postinfarto de miocardio). La disincronía del VI afecta el rendimiento sistólico y diastólico de ambos ventrículos. Yamaguchi y colaboradores determinaron que la contracción del VI contribuye al 20$40 \%$ de la presión sistólica del VD y la contracción del VD contribuye a 4-10\% de la presión sistólica del VI. 22,23

Ocupar un espacio pericárdico y compartir un septum afecta el lusitropismo ventricular. Con espacio limitado para expandirse, el aumento del llenado del ventrículo disminuye el cumplimiento diastólico del otro. Esto es evidente en los casos de embolia pulmonar con falla del VD en la que la dilatación masiva provoca el colapso del

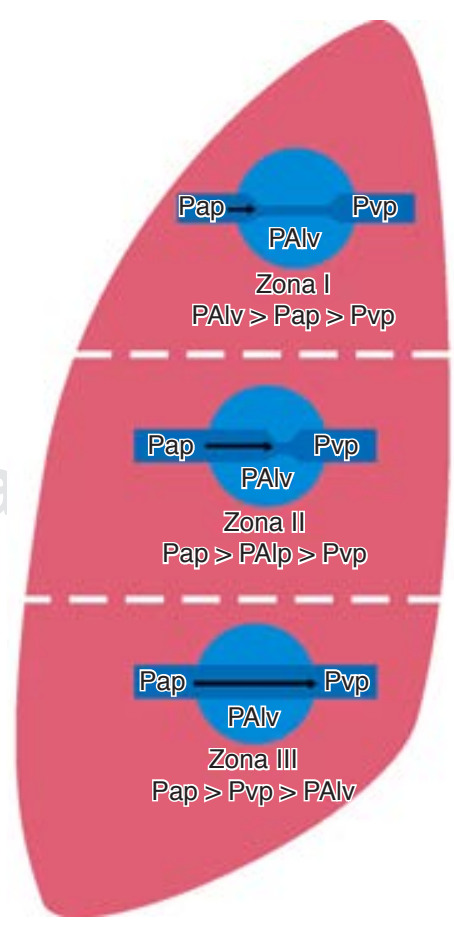

Figura 4:

Zonas de West. PAlv = presión alveolar; Pap = presión de la arteria pulmonar; Pvp = presión venosa pulmonar. 
volumen diastólico final del VI. La inspiración espontánea, al aumentar el retorno venoso y el volumen al final de la diástole del VD también causa cambios similares, aunque notablemente menos impresionantes, en el volumen diastólico del VI a lo largo del ciclo ventilatorio, independientemente de la presión de llenado del VI. A lo largo de la suma de latidos del corazón, el volumen promedio del VD debe ser igual al volumen del VI, pero existen variaciones importantes latido a latido, causadas por el efecto de la presión intratorácica en ambos ventrículos. En condiciones normales, la vasculatura pulmonar de baja elastancia y alta capacitancia permite que la vasculatura pulmonar se adapte a las variaciones en el volumen del VD sin mucho cambio en la presión de la arteria pulmonar. Así, la respiración espontánea aumenta el volumen del VD y disminuye el volumen sistólico del $\mathrm{VI}$, que se revierte con la exhalación, pero el gasto cardiaco en estado estable es relativamente constante. ${ }^{24,25}$

\section{COMPARACIÓN DE MODELOS DE VENTILACIÓN}

El efecto de la ventilación mecánica en el corazón y la hemodinámica se relacionan esencialmente en la forma en que cada modo de ventilación altera la PIT y el volumen pulmonar. Los diferentes modos de ventilación pueden afectar a los pacientes de manera similar si su impacto en la presión intratorácica y el volumen pulmonar es similar. Esto es cierto a pesar de marcadas diferencias en las formas de onda o las diferencias en la asistencia respiratoria total o parcial siempre que los volúmenes tidales y la PEEP se mantengan similares. La ventilación con control presión se ha comparado con la ventilación controlada por volumen que demuestra gasto cardiaco sin cambios si el volumen tidal es igual y el gasto cardiaco es elevado si el volumen tidal es bajo. ${ }^{1}$

Durante la ventilación mecánica invasiva el flujo de aire que se entrega al paciente determina una presión o volumen establecido al momento de programar el ventilador. Esto produce un aumento de la presión intratorácica que es mayor durante la inspiración, disminuyendo hasta la presión en reposo durante la espiración, la cual es pasiva. Lo anterior determina que durante las fases de la respiración la presión intratorácica sea mayor que la atmosférica. Esta presión positiva se transmite a todas las estructuras intratorácicas, lo que produce efectos en la presión intratorácica, aumentándola sobre las estructuras vasculares. A esto debemos sumar la presión positiva al final de la espiración, que eleva la presión intratorácica en ambas fases de la ventilación en la misma cuantía. ${ }^{1,3}$

Es así como la ventilación con presión positiva influirá en la precarga y postcarga de ambos ventrículos (Tabla 1). A nivel del VD la ventilación con presión positiva determina un aumento de la presión intratorácica, lo que eleva la presión en la $A D$, disminuyendo
Tabla 1: Ventilación a presión positiva e interacciones cardiopulmonares.

\begin{tabular}{ll}
\hline Disminuye precarga & Aumenta postcarga \\
\hline Ventrículo derecho & \\
Disminuye retorno venoso & Aumenta RVP \\
Disminuye VDFVD & Disminuye flujo pulmonar \\
Ventrículo izquierdo & \\
Disminuye llenado de Al & Disminuye PTVizq \\
Disminuye VDFVI & Aumenta VE y GC \\
\hline
\end{tabular}

RVP = resistencia vascular pulmonar; VDFVD = volumen diastólico final de ventrículo derecho; $\mathrm{Al}=$ aurícula izquierda; $\mathrm{VDFVI}=$ volumen diastólico final de ventrículo izquierdo; PTVizq = presión transmural del ventrículo izquierdo; VE = volumen expulsivo; $\mathrm{GC}$ = gasto cardiaco; $\mathrm{Al}$ = aurícula izquierda.

el gradiente de presión para el retorno venoso, lo que determinará una reducción del llenado de la $A D$ y por consecuencia del VD. A nivel de la postcarga del VD, la ventilación con presión positiva determina un aumento del volumen pulmonar con la consecuente elevación de la presión alveolar, lo que produce un incremento de la resistencia vascular pulmonar, aumentando la postcarga del VD y disminuyendo el flujo sanguíneo pulmonar. La precarga del VI se ve afectada como ya se comentó, la ventilación con presión positiva se reduce además de la precarga del VD, aumenta la postcarga de éste, resultando en una disminución del llenado de la aurícula izquierda, lo cual determina una baja del volumen diastólico final del VI. También durante la inspiración de la ventilación con presión positiva se produce un aumento del llenado de la aurícula izquierda por compresión del pool de sangre que se encuentra en los vasos alveolares; sin embargo, este efecto sólo se presenta en dos a tres ciclos. La postcarga del VI con la ventilación con presión positiva produce un efecto interesante, pues la ventilación con presión positiva determina un decremento de la presión transmural del VI por aumento de la presión intratorácica, lo que producirá por consiguiente una reducción de la postcarga del VI y en condiciones de euvolemia, un aumento del volumen expulsivo. En 25 pacientes con lesión pulmonar aguda, los efectos hemodinámicos de los modos de ventilación controlados por presión y por volumen fueron similares siempre que la presión de la vía aérea fuera similar en todos los modos. Singer y colaboradores demostraron en pacientes ventilados que una hiperinflación pulmonar, y no la presión de la vía aérea, disminuía el gasto cardiaco.,15

\section{CONTEXTOS CLÍNICOS}

La interacción corazón pulmón presenta alteraciones clínicamente relevantes en los pacientes bajo ventilación mecánica que pueden provocar inestabilidad hemodinámica, y el conocimiento de esta interacción ofrece la posibilidad de predecir las reacciones que presentará un paciente en su sistema cardiovascular. ${ }^{10,26,27}$ 
El ventrículo derecho se ve particularmente influenciado por la ventilación mecánica debido a que su retorno venoso y precarga están reducidos, esto está mediado por la elevación de la presión pleural, mientras que la precarga está mecánicamente incrementada por una reducción de la presión transmural de la vasculatura pulmonar. En los pacientes que presentan SDRA, estos efectos se ven aumentados por la presencia de vasoconstricción pulmonar, hipoxemia e hipercapnia así como microtrombosis pulmonar, cambios en las zonas de West y desreclutamiento alveolar, todo esto provoca hipertensión pulmonar con peor pronóstico. A pesar de las estrategias de empleo de bajo volumen tidal que pueda ayudar a disminuir los efectos mecánicos en el VD debido a las presiones bajas en la vía respiratoria, la presencia de cor pulmonale agudo es aún altamente frecuente, alrededor de $25 \%$, y se le asocia a un mal pronóstico. El riesgo de desarrollar cor pulmonale agudo se vuelve más alto con una peor oxigenación, hipercapnia, altas presiones en el ventilador y neumonía como causa del SDRA. La posición prona conduce a una mejor función del VD al reclutar las áreas pulmonares dorsales, lo que resulta en una reducción de la postcarga del VD. $23-25,28-31$

El estado de choque se caracteriza por una entrega de oxígeno tisular deficitaria, dado en parte por un consumo tisular elevado de oxígeno o una alteración en su capacidad de utilización, entre otros, teniendo como resultado hipoxia tisular y necrosis celular. Ante situaciones de aumento de la demanda de oxígeno se producen ajustes fisiológicos neurohormonales que contribuyen a que, a pesar de este incremento, las células no sufran daño hipóxico. Esto se logra mediante el aumento de la capacidad de extracción de oxígeno o el incremento de la producción de hemoglobina. Si se supera la entrega de oxígeno tisular crítico, aparece como compensación la redistribución del porcentaje de gasto cardiaco para los diferentes órganos, como la disminución de éste para órganos secundarios en favor de los más nobles, lo cual se logra por modificación de las resistencias vasculares sistémicas regionales. Con relación a estos mecanismos el gasto cardiaco limitado durante el choque, el diafragma que representa menos de $5 \%$ de la demanda de oxígeno y recibe menos de $3 \%$ del gasto cardiaco, se ve afectado por el aumento de la carga respiratoria secundaria a la acidosis metabólica durante la respiración espontánea, incrementando su consumo progresivamente, llegando a valores elevados hasta de $50 \%$ de la demanda de oxígeno total, lo que determina un aumento de la perfusión hacia este órgano en detrimento de los órganos más nobles. Este fenómeno tiene un límite, lo cual derivará en agotamiento muscular, terminando en paro respiratorio; todo esto asociado al trabajo respiratorio aumentado, y el uso de presiones negativas cada vez mayores exacerba los efectos de la presión intratorácica en la precarga del VI al disminuir el flujo desde las venas pulmonares, además de contribuir a menor distensibilidad del VI por desplazamiento septal, reduciendo el gasto cardiaco del VI, el cual empeora más por la disminución de la postcarga del VI, lo que puede determinar presión arterial baja, sobre todo diastólica, que compromete la perfusión cardiaca. ${ }^{3}$

Por lo anterior, el uso de variabilidad de presión de pulso es una indicación en pacientes en estado de choque, pues baja la demanda de oxígeno diafragmático al disminuir el trabajo respiratorio, redirigiendo el gasto cardiaco a órganos blanco, además permite mejorar el llenado del AI de forma transitoria y reduce la postcarga del $\mathrm{VI}$, condicionando un aumento del volumen eyectivo y con ello un mejor gasto cardiaco. Se deberá tener en consideración no emplear presiones medias de la vía aérea elevadas, pues aumentan la resistencia vascular pulmonar pudiendo disminuir el retorno venoso de la aurícula derecha, por lo que siempre se debe mantener una volemia adecuada con base en predictores de respuesta a volumen, para ello la variabilidad de presión de pulso mayor de $13 \%$ y la variación del peak de velocidad del flujo aórtico en más de $12 \%$ durante el ciclo respiratorio se consideran predictores adecuados de respuesta a volumen en adultos. ${ }^{3,4}$

En los casos de falla cardiaca sistólica del VI, tanto el volumen eyectivo como el gasto cardiaco se encuentran disminuidos. La variabilidad de presión de pulso reduce la postcarga del $\mathrm{VI}$, pero además permite que baje el consumo de oxígeno de la musculatura respiratoria y miocárdica. Además, en pacientes en ventilación espontánea que evolucionan a edema agudo pulmonar secundario a disfunción miocárdica, la presión negativa elevada puede disminuir aún más el gasto cardiaco del VI, razón por la cual los pacientes presentan una respuesta favorable frente al uso de variabilidad de presión de pulso, ya que baja la presión transmural sistólica del $\mathrm{VI}$, reduciendo la postcarga. Adicionalmente la disminución de la carga sobre la bomba respiratoria y cardiaca desencadena una reducción de la actividad del sistema nervioso simpático, lo que disminuirá la postcarga ventricular, siendo otro mecanismo por el que mejora el gasto cardiaco con el empleo de la variabilidad de presión de pulso. Por lo anterior, se sugiere el uso de inotrópicos y vasodilatadores como apoyo previo a la extubación ${ }^{3,4}$ en los pacientes con insuficiencia cardiaca, en quienes se decide suspender la variabilidad de presión de pulso para pasar a modo espontáneo y en quienes aumentará la postcarga ventricular izquierda por elevación de la presión transmural en el VI.

En los casos de patología pulmonar obstructiva grave, se entiende que tienen un aumento grave de la resistencia de la vía aérea generando, por tanto, presión intratorácica extremadamente negativa para lograr el ingreso del flujo del aire. Lo anterior además activa el 
sistema nervioso simpático y el sistema renina angiotensina aldosterona, lo que contribuye al aumento de la postcarga biventricular. Por otra parte, hay un incremento del retorno venoso de la AD. Estos cambios condicionan un aumento del llenado de la cavidad ventricular derecha, lo que desplaza el septum interventricular, limitando de esta forma el llenado del VI y con ello el volumen expulsivo y el gasto cardiaco correspondiente. Esta variación en la presión intratorácica determinará un aumento de la postcarga del $\mathrm{VI}$, secundario a una elevación de la presión transmural del ventrículo, lo que contribuirá a bajar el gasto cardiaco final del VI. De esta manera se produce una exageración en el decremento de la presión sistólica que ocurre dentro de un ciclo respiratorio, por sobre los valores esperados, llegando en ocasiones a $30 \mathrm{mmHg}$ o más, lo que se conoce como pulso paradójico. En estos pacientes el empleo de la variabilidad de presión de pulso contribuye a la disminución del efecto final en el gasto cardiaco, ya que al aplicar presiones positivas en la vía aérea se atenúa el efecto del desplazamiento del septum y se produce una reducción de la postcarga ventricular izquierda, lo que como efecto neto mejora el gasto cardiaco. Esto tiene que ir de la mano de un adecuado aporte de volumen para mantener la volemia, ya que estas presiones positivas afectarán el retorno venoso hacia la $A D$, lo que determina una precarga derecha disminuida, misma que puede anular los beneficios obtenidos de la variabilidad de presión de pulso sobre el gasto cardiaco. ${ }^{3}$

\section{CONCLUSIONES}

La ventilación es un fenómeno ubicuo y sus efectos en la función cardiovascular son un resultado obligatorio. Al comprender las simples determinantes individualizadas de sus interacciones, es posible entender las presentaciones más complejas de los modos ventilatorios avanzados, los niveles de insuficiencia cardiovascular y pulmonar y cómo interpretar sus hallazgos, con el fin de tratar a estos pacientes de la manera más eficiente para minimizar las interacciones perjudiciales entre el corazón y el pulmón, al mismo tiempo que preservar las beneficiosas.

\section{BIBLIOGRAFÍA}

1. Mahmood SS, Pinsky MR. Heart-lung interactions during mechanical ventilation: the basics. Ann Trans/ Med. 2018;6(18):349.

2. Sette P, Dorizzi RM, Azzini AM. Vascular access: an historical perspective from Sir William Harvey to the 1956 Nobel prize to André F. Cournand, Werner Forssmann, and Dickinson W. Richards. J Vasc Access. 2012;13(2):137-144.

3. Castillo-Moya A, Del Pozo Bascuñan P. Cardiopulmonary interactions: from physiology to clinic. Rev Chil Pediatr. 2018;89(5):582-591.

4. Bronicki R, Penny D. Cardiopulmonary interactions. En: da Cruz E, Ivy D, Jaggers J. Pediatric and Congenital Cardiology, Cardiac
Surgery and Intensive Care. London: Springer-Verlag; 2014; 161: 933-953.

5. Shekerdemian L, Bohn D. Cardiovascular effects of mechanical ventilation. Arch Dis Child. 1999;80(5):475-480.

6. Magder S. Bench-to-bedside review: An approach to hemodynamic monitoring--Guyton at the bedside. Crit Care. 2012;16(5):236

7. Magder S. Volume and its relationship to cardiac output and venous return. Crit Care. 2016;20:271.

8. Moller PW, Winkler B, Hurni S, Heinisch PP, Bloch A, Sondergaard $S$, et al. Right atrial pressure and venous return during cardiopulmonary bypass. Am J Physiol Heart Circ Physiol. 2017;313(2):408-420.

9. Tyberg JV, Taichman GC, Smith ER, Douglas NW, Smiseth OA, Keon WJ. The relationship between pericardial pressure and right atrial pressure: an intraoperative study. Circulation. 1986;73(3):428-432.

10. Grübler MR, Wigger O, Berger D, Blöchlinger S. Basic concepts of heart-lung interactions during mechanical ventilation. Swiss Med Wkly. 2017;147:w14491.

11. Lansdorp B, Hofhuizen C, van Lavieren M, van Swieten $\mathrm{H}$, Lemson J, van Putten MJ, et al. Mechanical ventilation-induced intrathoracic pressure distribution and heart-lung interactions. Crit Care Med. 2014;42(9):1983-1990.

12. Kilburn KH. Cardiorespiratory effects of large pneumothorax in conscious and anesthetized dogs. J Appl Physiol. 1963;18:279283.

13. Nanas S, Magder S. Adaptations of the peripheral circulation to PEEP. Am Rev Respir Dis. 1992;146(3):688-693.

14. Magder SA, Lichtenstein S, Adelman AG. Effect of negative pleural pressure on left ventricular hemodynamics. Am J Cardiol. 1983;52(5):588-593.

15. Katira BH, Giesinger RE, Engelberts D, et al. Adverse heart-lung interactions in ventilator-induced lung injury. Am J Respir Crit Care Med. 2017;196(11):1411-1421.

16. van den Berg PC, Jansen JR, Pinsky MR. Effect of positive pressure on venous return in volume-loaded cardiac surgical patients. J Appl Physiol (1985). 2002;92(3):1223-1231.

17. Buda AJ, Pinsky MR, Ingels NB Jr, Daughters GT 2nd, Stinson EB, Alderman EL. Effect of intrathoracic pressure on left ventricular performance. N Engl J Med. 1979;301(9):453459.

18. Vieillard-Baron A, Matthay M, Teboul JL, Bein T, Schultz $M$, Magder S, et al. Experts' opinion on management of hemodynamics in ARDS patients: focus on the effects of mechanical ventilation. Intensive Care Med. 2016;42(5):739-749.

19. West JB. Respiratory physiology the essentials. 9th edition. Philadelphia: Lippincott Williams \& Wilkins; 2012.

20. Morimont P, Lambermont B, Ghuysen A, Gerard P, Kolh P, Lancellotti $P$, et al. Effective arterial elastance as an index of pulmonary vascular load. Am J Physiol Heart Circ Physiol. 2008;294(6):2736-2742.

21. Matthews JC, McLaughlin V. Acute right ventricular failure in the setting of acute pulmonary embolism or chronic pulmonary hypertension: a detailed review of the pathophysiology, diagnosis, and management. Curr Cardiol Rev. 2008;4(1):49-59.

22. Jardin F, Genevray B, Brun-Ney D, Bourdarias JP. Influence of lung and chest wall compliances on transmission of airway pressure to the pleural space in critically ill patients. Chest. 1985;88(5):653-658.

23. Vieillard-Baron A, Loubieres Y, Schmitt JM, Page B, Dubourg $\mathrm{O}$, Jardin F. Cyclic changes in right ventricular output impedance during mechanical ventilation. J Appl Physiol (1985). 1999;87(5):1644-1650.

24. Magder S, Guerard B. Heart-lung interactions and pulmonary buffering: lessons from a computational modeling study. Respir Physiol Neurobiol. 2012;182(2-3):60-70.

25. Taylor RR, Covell JW, Sonnenblick EH, Ross J Jr. Dependence of ventricular distensibility on filling of the opposite ventricle. Am J Physiol. 1967;213(3):711-718.

26. Vieillard-Baron A, Schmitt JM, Augarde R, Fellahi JL, Prin S, Page B, et al. Acute cor pulmonale in acute respiratory distress syndrome submitted to protective ventilation: incidence, clinical 
implications, and prognosis. Crit Care Med. 2001;29(8):15511555.

27. Pinsky MR. Functional haemodynamic monitoring. Curr Opin Crit Care. 2014;20(3):288-293.

28. Duggan M, McCaul CL, McNamara PJ, Engelberts D, Ackerley C, Kavanagh BP. Atelectasis causes vascular leak and lethal right ventricular failure in uninjured rat lungs. Am J Respir Crit Care Med. 2003;167(12):1633-1640.

29. Bull TM, Clark B, McFann K, Moss M; National Institutes of Health/National Heart, Lung, and Blood Institute ARDS Network. Pulmonary vascular dysfunction is associated with poor outcomes in patients with acute lung injury. Am J Respir Crit Care Med. 2010;182(9):1123-1128.

30. Brower RG, Matthay MA, Morris A, Schoenfeld D, Thompson BT, Wheeler A; Acute Respiratory Distress Syndrome
Network. Ventilation with lower tidal volumes as compared with traditional tidal volumes for acute lung injury and the acute respiratory distress syndrome. N Engl $J$ Med. 2000;342(18):1301-1308.

31. Mekontso Dessap A, Boissier F, Charron C, Begot E, Repesse X, Legras A, et al. Acute cor pulmonale during protective ventilation for acute respiratory distress syndrome: prevalence, predictors, and clinical impact. Intensive Care Med. 2016;42(5):862-870.

Correspondencia:

Humberto Ochoa Salmorán

E-mail: hochoas2528@gmail.com

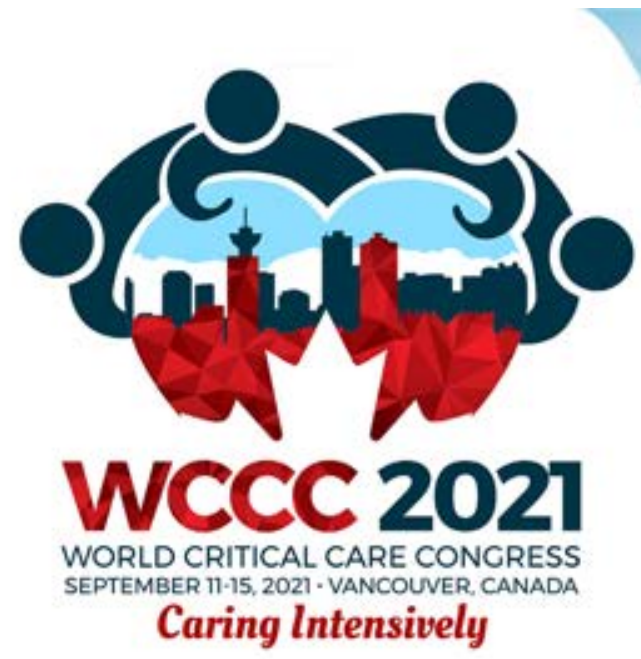

Meeting is hosted by:

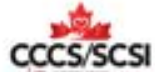

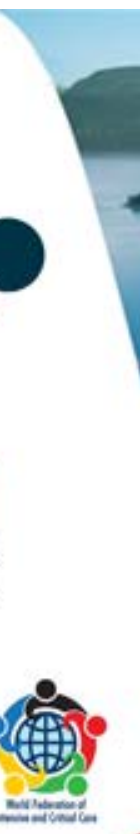
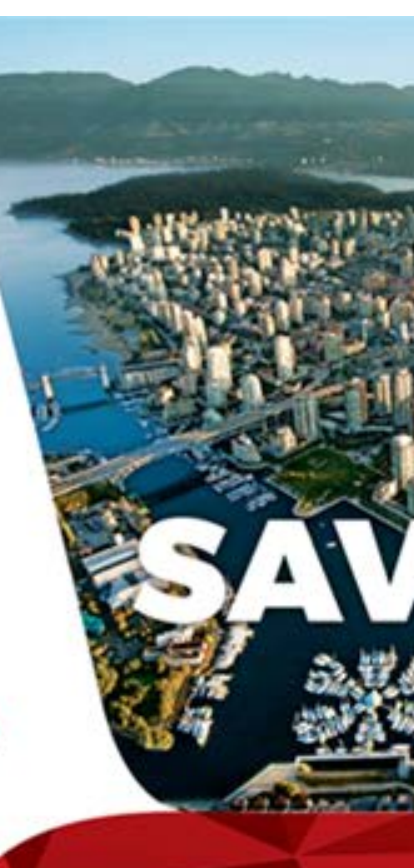

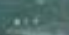
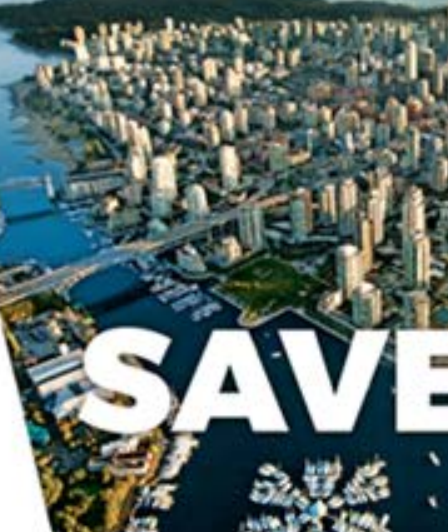

sive
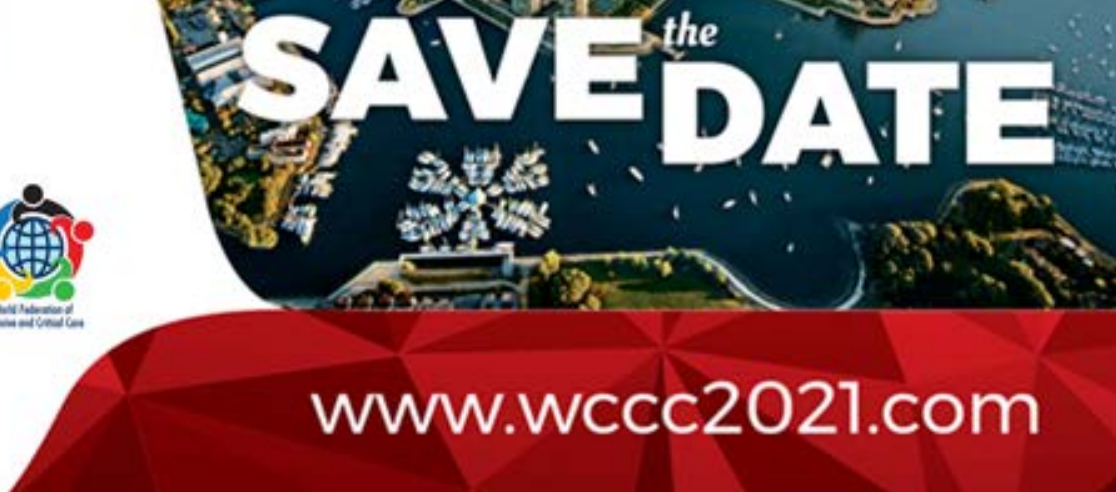